MARCELO A. ESPINOSA MARTÍNEZ

\title{
LA ARQUITECTURA DEL HOYO. UNA LECTURA EMPIRICA DEL HOYO
}

\author{
El autor es Arquitecto (ITESM, México), Realizó estudios de Arquitectura en el SCI-Arc, California y de Maestría en \\ Diseño Arquitectónico y Urbano en el DRL, Londres. Es Master en Arquitectura y Cultura Urbana (UPC, Barcelona) y \\ actualmente realiza estudios de Doctorado en el Architectural Association (Londres). Es profesor de cátedra en Diseño \\ Arquitectónico y Urbano en el Departamento de Arquitectura del ITESM (México).
}

\begin{abstract}
RESUMEN. El texto busca entender el concepto del "hoyo" dentro de la teoría arquitectónica, rescatando a esta categoría del papel que juega dentro del discurso filosófico para regresarlo a la materialidad dentro de la teoría arquitectónica. La filosofía a tratado el estatus del hoyo como un problema teórico y siempre lo a confinado en términos de su negatividad y negación, dentro de los problemas de la nada. En este texto los argumento filosóficos concernientes con la "nada" son considerados solamente con la finalidad de ser transformados en conceptos positivos dentro de la teoría arquitectónica. Este ensayo se propone abrir el argumento para una discusión posterior. y presentar acercamientos hacia cuales podrían considerarse "hoyos" en el entorno físico. También analiza, brevemente, la cuestión empírica de los hoyos descritos por la arquitectura, mostrando que producen condiciones y efectos mucho más complejos de los que normalmente la arquitectura reconoce.
\end{abstract}

ABSTRACT. ARCHITECTURE OF HOLES. An empirical reading of holes.

This text tries to understand the concept of the hole from within architectural theory. It aims to rescue it from the role it plays in the philosophical discourse in order to return to the architectural theory as a material entity. Philosophy has always considered the status of the "hole" as a theoretical issue and always confined in terms of its negativity or to problems related to the notion of "nothingness". In this text, philosophical arguments concerning "nothing" are treated seriously, but only in so far as they may be transformed into positive concepts within the architectural theory.

This article aims to open this argument to further discussion by posing a series of encounters with what we might denote as holes in the physical realm. Also works briefly through the question of empirical holes described by architecture and the fine arts, showing that they produce far more complex conditions and effects that we have normally recognized in architecture.

\footnotetext{
"The emptiness, the void, is what does the vessel's holding. The empty space, this nothing of the jug, is what the jug is as the holding vessel...The vessel's thingness does not lie at all in the material of which it consists, but in the void that holds." Martin Heidegger, The Thing
}

\section{INTRODUCCIÓN}

El análisis teórico de los elementos más simples dentro de la arquitectura encuentran su base dentro de lo complejo del espacio. Lo que parece más elemental a veces se convierte en un problema de considerable complejidad. Un ejemplo de esto es una entidad como el "hoyo". Un "hoyo", a primera instancia, podría considerarse como un concepto elemental y empírico sin mérito para una investigación a detalle.

Al parecer, se solventa en una premonición dentro del marco común de la vida diaria, y de igual forma, parece no contemplar ningún interés para la teoría arquitectónica. Como podemos mover de un sentido común la 
cuestión que implica el "hoyo" para presentarlo como una cuestión inseparable de la arquitectura, pero a la vez resistente a los términos elementales dentro del análisis arquitectónico.

En este texto vamos a tratar de delinear ciertos puntos, los cuales nos permitan entender el concepto del "hoyo" dentro de la teoría arquitectónica. Dentro de esto nos encontramos con dos problemas: el primero es la categorización empírica del hoyo dada dentro de la especulación arquitectónica. El segundo problema es, que mientras la filosofía a tratado el estatus del hoyo como un problema teórico, siempre lo a confinado en términos de su negatividad, negación y dentro de los problemas de la nada. El objetivo de este ensayo es, rescatar esta categorización del hoyo del papel que juega dentro del discurso filosófico y regresarlo a la materialidad dentro de la teoría arquitectónica, no en términos denotando negatividad pero en un objeto arquitectónico positivo real. Claro que esto significa que, los argumento filosóficos concernientes con la "nada" deben de ser considerados seriamente, pero solamente hasta donde estos puedan transformarse en conceptos positivos dentro del "hoyo arquitectónico". Quizás no podamos cubrir por completo la noción del "hoyo" aquí, pero esto abrirá este argumento para discusión posterior. Al mismo tiempo, vamos a presentar acercamientos los cuales podríamos considerar como "hoyos" en el entorno físico. También analizaremos, brevemente, la cuestionante empírica de los hoyos descritos por la arquitectura, mostrando que producen condiciones y efectos mucho más complejos de los que normalmente la arquitectura reconoce.

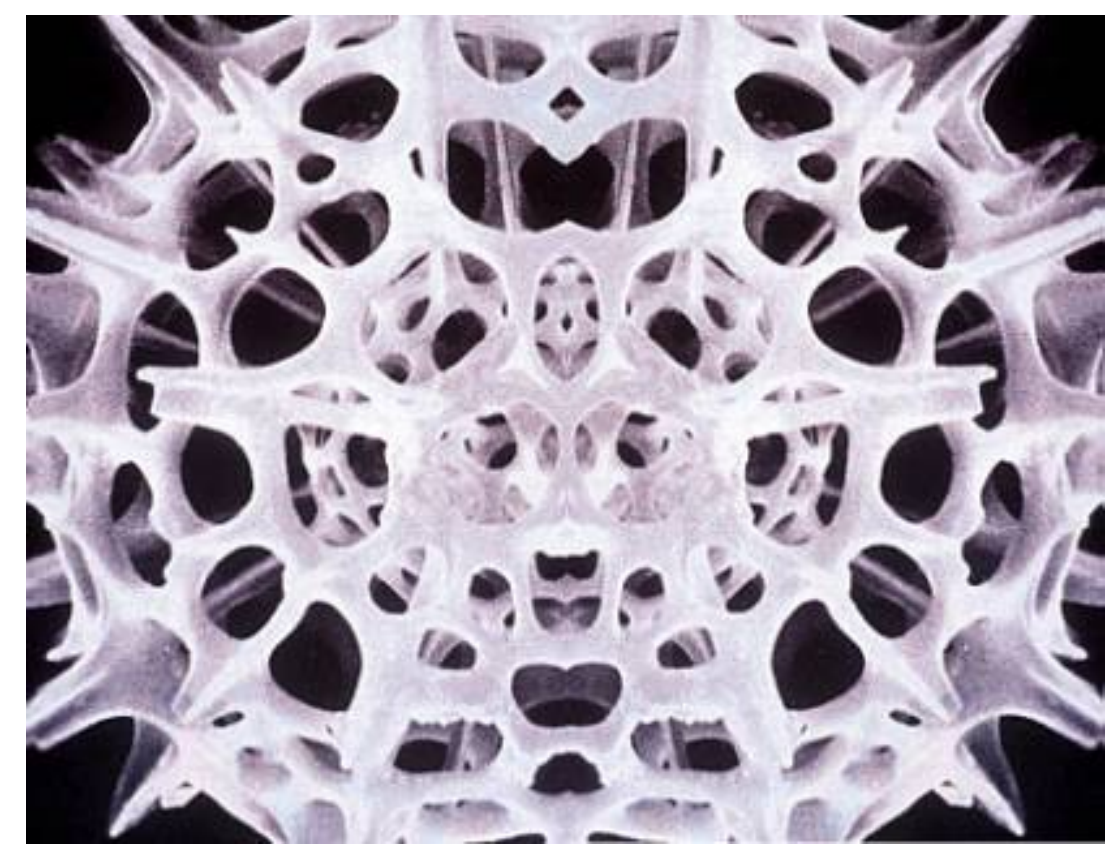

\section{EL CONCEPTO DE LA NADA O EL HOYO COMO PUNTO INCIAL}

Tratando de pensar en un hoyo representa la particularidad de situarnos dentro de una dualidad de pensamiento, entre lo material y lo inmaterial. Algo que se justifica en no estar presente pero capaz de afectarnos mentalmente a través de la percepción. Existen diferentes líneas de estudio que involucran interiormente el pensar en algo, a la vez, abstracto y como una entidad física. La investigación de filosofías concernientes al problema de la nada, o al vacío, las cuales claramente tienen un gran espectro dentro de la metafísica y su historia, pueden presentar nuestro primer acercamiento hacia el concepto del hoyo. Debido al espacio de este texto, solamente trataremos de indicar ciertos puntos en nuestro argumento, los cuales nos permitirán llegar a nuestro principal interés: la noción de los hoyos en la realidad física. De alguna manera, el hoyo es tratado en la ontología tradicional dentro del caso del "no-ser". Por lo tanto, la sustancia de su precedente esta claramente denotada por los argumentos tanto canónicos como modernos del ser y la nada. Si tratamos de ubicarnos dentro de un pensamiento existencialista, la noción del "no-ser" o la "nada" es la serie de faltas, ausencias, vacíos, etc. que nos rodean en el mundo. No tienen un estatuto "objetivo" afuera en la realidad. De cierta manera, si 
volteamos hacia algo dentro de una realidad objetiva, estos "no-ser" o faltantes hacen una respuesta negativa apropiada. Vienen a representar la constante matemática donde para cada positivo siempre existe su parte negativa, es él "algo" faltante dentro del "ser" objetivo. En otras palabras y acorde con el escritor y filósofo francés Jean-Paul Sartre, estas pequeñas "nadas" en el mundo —faltantes, ausencias, fallas, destrucciones, etc. - todas se presentan, eso es, aparecen fenomenológicamente hacia mi siendo algo que yo descubro o aprendo. Aparecen como «cosas» objetivas, cosas de las cuales se pueden hacer un mal juicio, pero a final, entidades perceptibles dentro del mundo material. Todas estas características - la posibilidad de aprender, de descubrir, de arriesgar, de la objetividadson características concernientes a la percepción.

La distinción entre él "ser" y el "no-ser" es, ontológicamente hablando, la división entre entidades concretas y las abstractas. Dentro de una visión común, las entidades abstractas son entendidas como no-seres en el espacio físico y en tiempo, carente de extensión física y de la cualidad de cambiar. En el mundo real o en el "mundo de las cosas", "cosa", en el sentido mas general, es intercambiable por el concepto "entidad" o "ser" y es aplicable a cualquier objeto cuya existencia es entendida por un sistema ontológico. De cualquier manera, es mucho más común en términos filosóficos y metafísicos el empleo del concepto de "cosa" en un sentido mucho mas restrictivo, el cual puede ser substituido por el termino "objeto", el cual posee propiedades, se constituye en relación con otros y contiene la particularidad de cambio, los cuales constituyen eventos. Vamos a tratar de "trabajar a través" del termino del "hoyo", de forma que podamos configurar un análisis breve y conciso acerca de la percepción, constitución y potencialidad del concepto de la "nada", alrededor de lo que podríamos considerar la carencia de espacio en el espacio.

Esto podría introducir una nueva dirección hacia un paradigma el cual re-invente, dentro de su potencial significante, pensamiento e ideas dentro de la interioridad del "hoyo", y más que todo, porque hemos clasificado este peculiar fenómeno como "hoyo".

\section{UNA PECULIAR LÓGICA FORMAL SOBRE HOYOS DENTRO DE LA REALIDAD ARQUITECTÓNICA}

Conforme avanzamos sobre nuestro argumento, no debemos perder la dirección y vernos dirigidos completamente por una visión existencialista o filosófica. Pero, debemos también reconocer que esto podría abrir un nuevo argumento dentro del discurso contemporáneo de la arquitectura por medio de que podamos establecer conclusiones actuales sustentadas sobre la noción del hoyo y su papel en la producción de arquitectura.

La alegoría de la cueva empleada por Platón (en La República, libro VII), mas que ser un ejemplo de razonamiento puro sobre los objetos que constituyen el mundo, es una intrínseca metáfora de un espacio aislado interior. La cueva representa una relación actual entre el afuera y el adentro. Según Platón, el afuera esta constituido de "formas" ideales, los cuales se conforman de patrones basados en formas geométricas perfectas. En cambio, en el adentro, el oscuro y desconocido espacio de la cueva, es donde las formas del afuera se proyectan de manera distorsionada a través de sombras para convertirse en "otro" mundo, el mundo del "adentro".

Estas percepciones interiores y exteriores son completamente diferentes una de la otra. Pero, ¿qué es este espacio del "adentro", qué tipo de cualidades este espacio contiene? Dentro de otro punto de vista, en sus conferencias sobre estética, Hegel se refiere a las construcciones subterráneas de la antigüedad, no tanto formalmente, pero sí dentro de la idea de la eliminación de materia. Para él, — dentro de las excavaciones, no es tanto el concepto de construcción positiva pero más en el remover lo negativo-. Él considera a las cuevas como una expansión de la materialidad en orden para crear espacio, mas que verlo como una limitante, o un recinto limitado. Algo interesante que surge de este concepto es la -expansión espacial- producida dentro de este proceso. ¿Cómo esta noción de construcción-a-través-de-excavación, o estos espacios \pm cavernosos, se traducen en arquitectura o en el ambiente construido? 


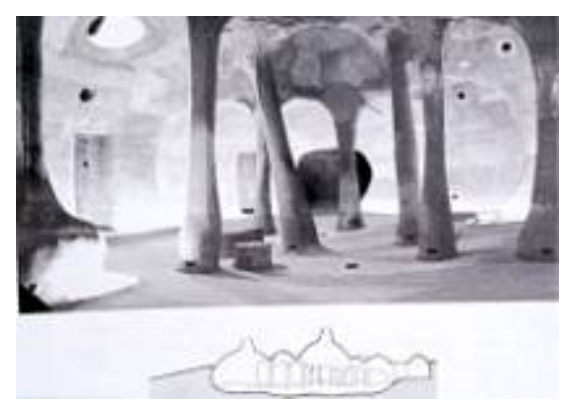

Foto 2. La alegoría de la cueva empleada por Platón mas que ser un ejemplo de razonamiento puro sobre los objetos que constituyen el mundo, es una intrínseca metáfora de un espacio aislado interior.

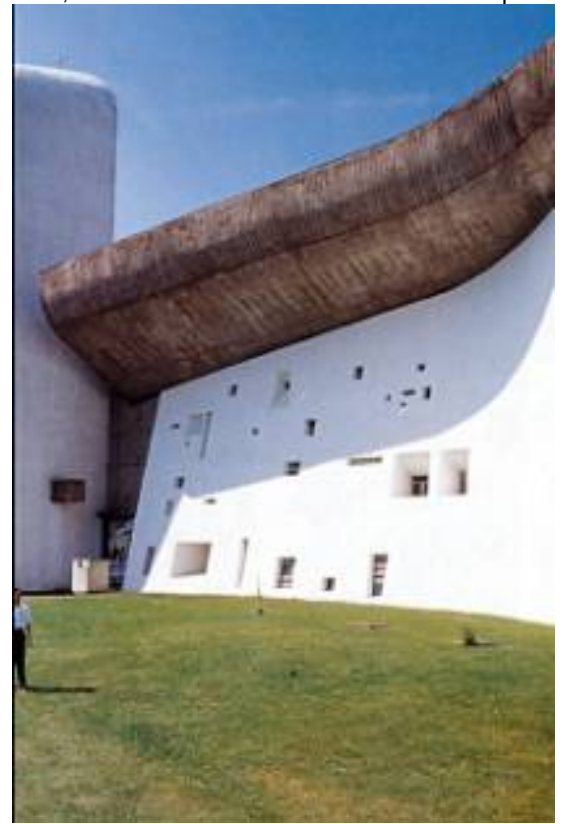

Foto 3. Casi al final de su carrera, Le Corbusier abrió un nuevo concepto dentro del contexto de la arquitectura moderna, el desplazamiento del fondo dentro del concepto de figura/fondo.

Regresemos al termino del "hoyo" dentro de la arquitectura, y tratemos de instrumentalizar la noción del hoyo a través de diferentes acercamientos dentro de la realidad física. De un cierto tiempo a la fecha, la producción de la arquitectura contemporánea ha tratado de establecer parámetros para la implementación de lo que pudiéramos considerar un «hoyo espacial». Antes de plantear "estos" lugares- de contacto, quisiera presentar un par de puntos importantes para nuestro argumento y para tenerlos como futuras referencias. Dentro del Movimiento Moderno, la arquitectura estaba preocupada en una configuración del -espacio abierto- en el gran espacio libre. A un lado de esta propuesta, y casi al final de su carrera, Le Corbusier, una de las principales figuras detrás de esta idea de la planta libre, abrió un nuevo concepto dentro del contexto de la arquitectura moderna, el desplazamiento del fondo dentro del concepto de figura/fondo, o de la superficie de sustento. Para él, esta superficie de sustento, o "terreno", no existía en el abajo solamente, sino también en el arriba: en la fachada o en la cubierta superior, convirtiéndose en una extensión de esta superficie de sustento. "Terreno" no era considerado como un elemento estático sino como un elemento dinámico. Para el propósito de nuestro argumento, asumamos que el terreno es espacio. Para esto, necesitamos localizar y re-pensar el concepto de "terreno" o de la superficie de sustento. Esto nos ayudara a poder re-direccionar la identificación de "hoyos" tanto en términos fenomenológicos como físicos. Si concordamos que la superficie de sustento, o "terreno", es una entidad material donde una lectura toma lugar, tenemos que soportar un desplazamiento de la superficie que compone el "terreno". En otras palabras, cuando percibimos un hoyo en una superficie es porque la superficie esta ahí, el hoyo existe en algo material. La construcción física de un "hoyo" en una superficie involucra, lo que Hegel llamo, la "lógica de excavación". Esta lógica estaba basada básicamente en un proceso de substracción. Se crea espacio a través del proceso de remover materia de un objeto o entidad. Este proceso involucra la 
substracción de materia "positiva" y con esto creando espacio con materia "negativa". En este sentido, tenemos una entidad "negativa" consumiendo espacio. Con esto podemos establecer nuevos parámetros para el modo de operación de la superficie de sustento, o el "terreno", de forma que podamos entender mejor el concepto del "hoyo" en la arquitectura.

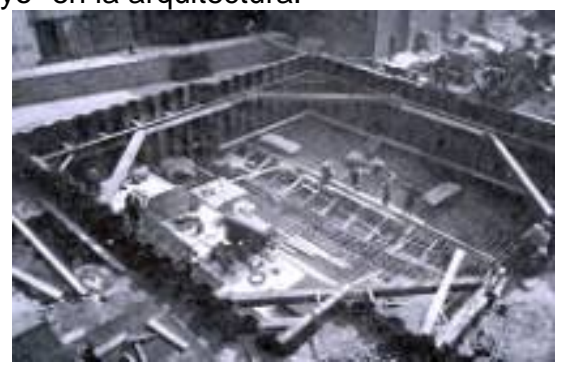

Foto 4. La construcción física de un "hoyo" en una superficie involucra, lo que Hegel llamo, la "lógica de excavación".

\section{REPRESENTACIÓN DEL HOYO}

En una detallada inspección, podríamos encontrar un numero considerable de ejemplos en los últimos treinta años dentro de la arquitectura los cuales han tratado de trabajar con este fenómeno o esta operación de substracción, o proceso de excavación como método para crear hoyos. Proyectos recientes como: el Museo Long Beach (1986), proyecto generado a través de trazos resultantes de un proceso de excavación o el edificio DAAP en Ohio (1996) configurado a través de un proceso de creación basado en el vacío, ambos proyectos del arquitecto Peter Eisenman.

El proyecto para la Bibliotheque de France del arquitecto Rem Koolhaas/OMA, introduce la noción de una cavidad interna como una discontinuidad programática dentro del edificio; proyectos de Massimiliano Fuksas o de Will Alsop, donde esta noción del "hoyo-espacial" toma parte central en medida que representa una alteración visual de nuestro espectro de lectura espacial; o donde el "hoyo" asume el carácter de una entidad parasítica, como en el proyecto del arquitecto Toyo Ito en la Mediatheque en Sendai, Japón (1999). Podríamos considerar estos proyectos como especulaciones formales iniciales de la noción del "hoyo-espacial" en la arquitectura o lo que podríamos llamar la "instrumentalización del hoyo". En cierta medida, esto nos permitiría establecer directrices para formar conclusiones para el replanteamiento y repensamiento del potencial de estas entidades dentro de la arquitectura. Pero, de igual forma se debe tener cuidado, ya que no hablamos de aberturas, sino de construcciones actuales de espacio vacío ilusorio.
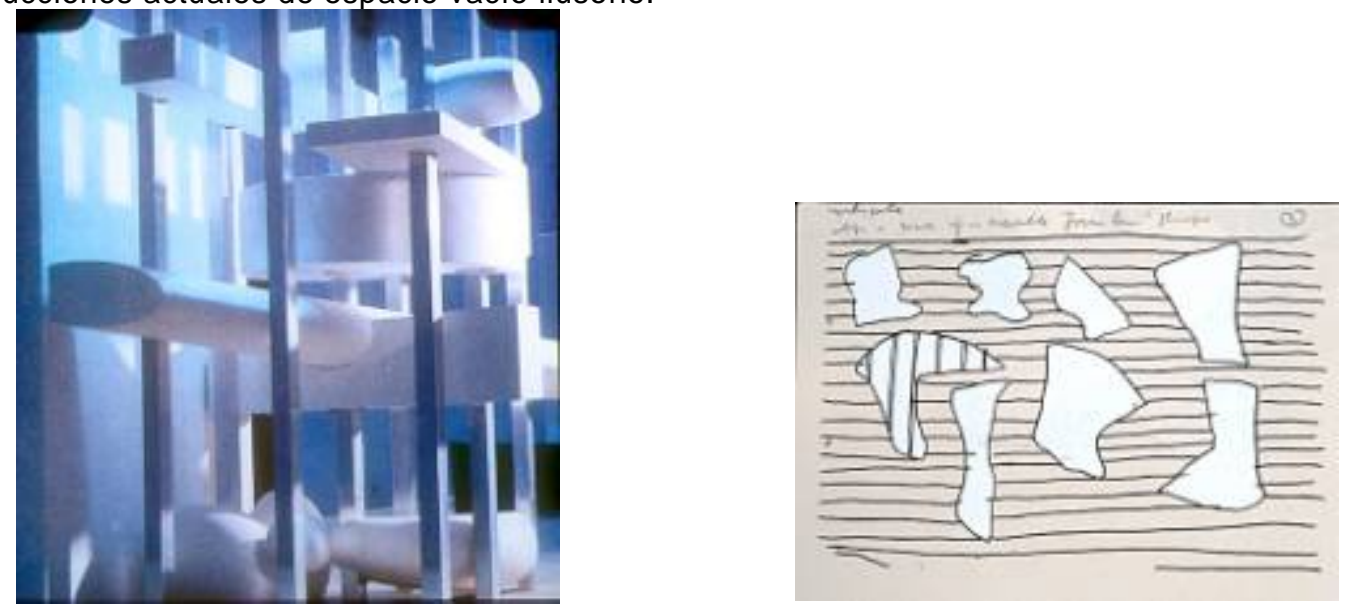

Fotos 5 y 6. El proyecto para la Bibliotheque de France, del arquitecto Rem Koolhaas/OMA, introduce la noción de una cavidad interna como una discontinuidad programática dentro del edificio.

Tratemos de usar esta noción del "hoyo" dentro del proyecto de la Bibliotheque de France, realizado por el arquitecto Rem Koolhaas. Un bloque sólido de información, un vasto contenedor de conocimiento, una bodega para todo el tipo de recolección de la memoria: libros, videos, películas, etc. 
Sin entrar en las implicaciones de como pensar sobre el concepto de un a biblioteca, el proyecto para la nueva biblioteca nacional de Francia de Koolhaas se compromete en una forma diferente de pensar sobre el funcionamiento del programa dentro de una nueva configuración espacial.

En relación con el almacenamiento y espacio de lectura común de una biblioteca, el proyecto contiene cinco librerías autónomas (De la imagen móvil y sonido, Adquisiciones recientes, Referencia, Catalogo y de Investigación). En este contenedor de losas, estos espacios de acceso publico se definen como ausencia de lo construido, volúmenes de espacio los cuales son removidos de la masa de información, espacio que perfora espacio o espacio que "flota en la memoria". Estas ausencias se presentan como múltiples embriones flotando entre las losas, donde cada uno de estos se conecta de una manera particular con la infraestructura principal. Para Koolhaas, el programa de funciones consistía de una serie de elementos repetitivos, aburridos y previsibles para la mayor parte del edificio, pero de la misma forma contenía una serie de entidades únicas y especiales. Al aceptar la monotonía presente en la mayor parte de los componentes del edificio, o de cierta manera, la inhabilidad para trabajar de manera creativa en la invención de estas entidades especiales, el proceso de diseño abre en nuestro argumento la idea de pensar dentro de lo negativo. Lo negativo es, aquí, usado no en términos de lo no posible, sino en la manera en que el edificio se puede elaborar desde el interior, no sumando pero en substrayendo. La "caja sólida" estuvo pensada como -dada-, como la superficie de sustento o "terreno" que mencionamos anteriormente.

Los nuevos espacios públicos son generados simplemente en el hecho de no construir materialidad. Estaba comprendido una suficiente densidad de programa, un volumen programático fijo, donde en términos de Koolhaas, una simple ausencia de edificio dentro del edificio podría, dentro del mismo, configurar de una manera mucho mas rica y refinada las expectaciones del programa y eventos públicos. La aceptación de cierta fijación en la serie de losas de almacenamiento que incesantemente persisten en todo el edificio, pone en funcionamiento la acción de excavación, o de perforación de manera que puedan acomodar las nuevas bibliotecas, estas son excavadas del sólido de información. Para Koolhaas, dejando de "construir" edificio podía solucionar la organización de los diferentes componentes públicos del programa, y con esto, toda la lógica de construcción trabaja a través de la ausencia de "edificación".

"Estaba claro que al no concebir los elementos especiales del edificio como cosas o objetos que estuvieran que ser construidos laboriosamente pero como áreas que pudieran dejarse fuera del edificio -como negativos- de manera que esto permitiera experimentar con ciertas cualidades espaciales las cuales seria casi imposible de construir como positivos" (Rem Koolhaas, Precarious Entities, Anyone, Rizzoli, New York, 1991)

Michael Hays presenta el proyecto de la Biblioteca en su libro Teoría Arquitectónica desde 1968 (The MIT Press, 1998) y el se refiere a estas intervenciones provocadas por estos componentes espaciales como "hoyos", donde cada librería se convierte en una entidad con su propia lógica inherente, cada uno independiente del otro, independiente de la superficie exterior, y de las formas de constantes usuales de la arquitectura. De la misma manera, la ambición del proyecto de Koolhaas era la necesidad de librarse de las responsabilidades de la arquitectura, que para él, no puede sostener mas en la producción de la arquitectura contemporánea. La Biblioteca buscaba dentro de su interioridad, escapar de la lógica de la presencia, de manera que esta fuera sobre-escrita por la manifestación de la nada, o por la representación de la ausencia dentro de su misma concepción.

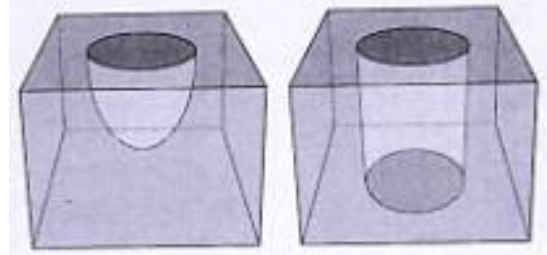

Foto 7. Roberto Casati y Achille C. Varzi describen a los hoyos como "entidades cognada", cosas que están juntas a otras, o en relación con otras.

Lo que hemos considerado como hoyos "reales", hasta ahora, han sido elaboraciones basadas en la noción de ausencias, cosas que no están presentes. Incluso en el sentido común, la distinción entre adentro y afuera está, hasta cierto grado, unida al concepto del hoyo. Pensar acerca de un hoyo, es de 
cierta medida, pensar y razonar sobre la forma de un "objeto", pero también de igual manera sobre la disposición de este para interactuar con otros objetos; de la forma de como es un hoyo o como es creado, como puede ser modificado, usado, destruido; y finalmente, acerca de la manera como un hoyo es percibido, identificado y re-identificado. En este sentido, podemos hablar de hoyos en la Arquitectura. No hoyos o espacios muertos dentro de la referencia histórica de la misma, sino de hoyos materiales dentro del objeto, en el "objeto arquitectónico". Existen objetos los cuales su función radica en sus perforaciones. El objeto arquitectónico, de manera perceptual, funcional y de escala diferente, parece ser una institución primaria basaba en perforaciones. Para analizar notaciones abstractas sobre hoyos, de forma que podamos relacionarlas a construcciones actuales dentro del arte y la arquitectura, es conveniente soportarnos en un nuevo acercamiento formal y de percepción hacia "hoyos" en la arquitectura. En su libro, "Hoyos y otras Superficialidades", (The MIT Press, 1994) Roberto Casati y Achille C. Varzi describen a los hoyos como una "entidades cognada", cosas que están juntas a otras, o en relación con otras. Los hoyos son particulares o individuos superficiales, en este sentido particulares dependientes: no pueden existir solos, necesitan un "anfitrión" donde puedan habitar.

En términos generales, estas entidades pueden ser descritas como depresiones superficiales, túneles o cavidades internas. Siempre que existe o nos encontramos con un hoyo hay una discontinuidad superficial que involucra una concavidad: uno puede "caerse" en un hoyo, pero uno no puede "chocar" con uno, te puedes esconder en un hoyo, pero no detrás de uno. Pero como este tipo de hoyos son generados y pueden ser utilizados dentro de la arquitectura. En relación con nuestro análisis sobre el proyecto de Koolhaas, como un ejemplo, y dentro del marco planteado por Casati y Varzi, los hoyos pueden ser considerados como cuerpos inma-teriales flotando en la superficie, alrededor o adentro del objeto. El hoyo, aquí, se convierte en una parte "superficial" del objeto, no depende solamente en estar en la superficie como una concavidad o internamente como una cavidad, ya que la simple existencia del mismo produce una relación con la superficie, la cual existe o ha sido creada. Cuando pensamos en "superficial", para los propósitos de nuestro argumento, debemos alejarnos de su fisicalidad y pensarlo mas en términos de "espacio". Las nuevas entidades, conformadas por las librerías especiales, actúan sobre el espacio como cavidades internas, perforando y consumiendo espacio.

Según la Mereología, los hoyos están "hechos" de cualquier materia pero son definidos por espacio. En este sentido, la materia de los hoyos es espacio, pero los hoyos son "cosas" que pueden estar compuestas de espacio solamente y no ser idénticos con otras regiones de espacio dentro del objeto. Los hoyos son claramente entidades escurridizas reconocibles. Son localizables en espacio y tiempo, de esta manera se pueden mover a través de las regiones de espacio que componen el objeto donde se ubican. Junto con este argumento, desarrollan un a condición parasítica dentro del objeto. El proyecto para la Mediateca de Sendai en Japón (1995) del arquitecto Toyo Ito, es un contenedor-caja objeto conformado por siete panel-losas de acero dispuestas en capas siguiendo la forma del contenedor. Estas losas son perforadas de manera vertical por doce mallas-tubulares de acero en forma de tubo. Estos túneles verticales internos se mueven a través de las losas y del espacio con una cierta autonomía en referencia al objeto, y al mismo tiempo, por su diferenciación parece dislocar su particular función. (Estructura, infraestructura, circulación vertical, etc.) La sensación de estabilidad parece contraponerse debido a su singular configuración de estructura trabajando como una malla tubular. Acorde con la lógica de los hoyos, estos pueden empezar con un cierto perfil, pero su configuración actual o total no puede ser percibida a primera instancia. Si uno piensa en un hoyo, uno siempre piensa en la forma o figura de algo. Un hoyo esta donde algo no esta.

Dentro del argumento de Casati y Varzi, "la complejidad morfológica del hoyo es espejada por la estructura topología de su -piel-." La piel es descrita como la parte de la superficie de un objeto la cual esta en contacto con el "contenido" del hoyo o de lo que esta compuesto el hoyo. La superficie de la piel es la primera parte del hoyo en ponerse en contacto con el ambiente del objeto, de esta manera la piel se convierte en la ultima zona de articulación entre lo que es arquitectura y lo que podría ser otra cosa, algo flotando entre lo inmaterial y la realidad material. Los hoyos se presentan entre dos condiciones, el "anfitrión" del hoyo, donde el hoyo se localiza y el "huésped" del hoyo, de lo que el hoyo esta hecho. Acorde con ellos, la identidad del hoyo no depende de la identidad de la materia en el interior (huésped), y al mismo tiempo no parece depender de la identidad del "anfitrión" del hoyo. Los hoyos son una "entidad sin identidad." Ellos pueden diferir, como tratamos de argumentar en los dos proyectos antes mencionados, de las cualidades espaciales del objeto donde residen. 

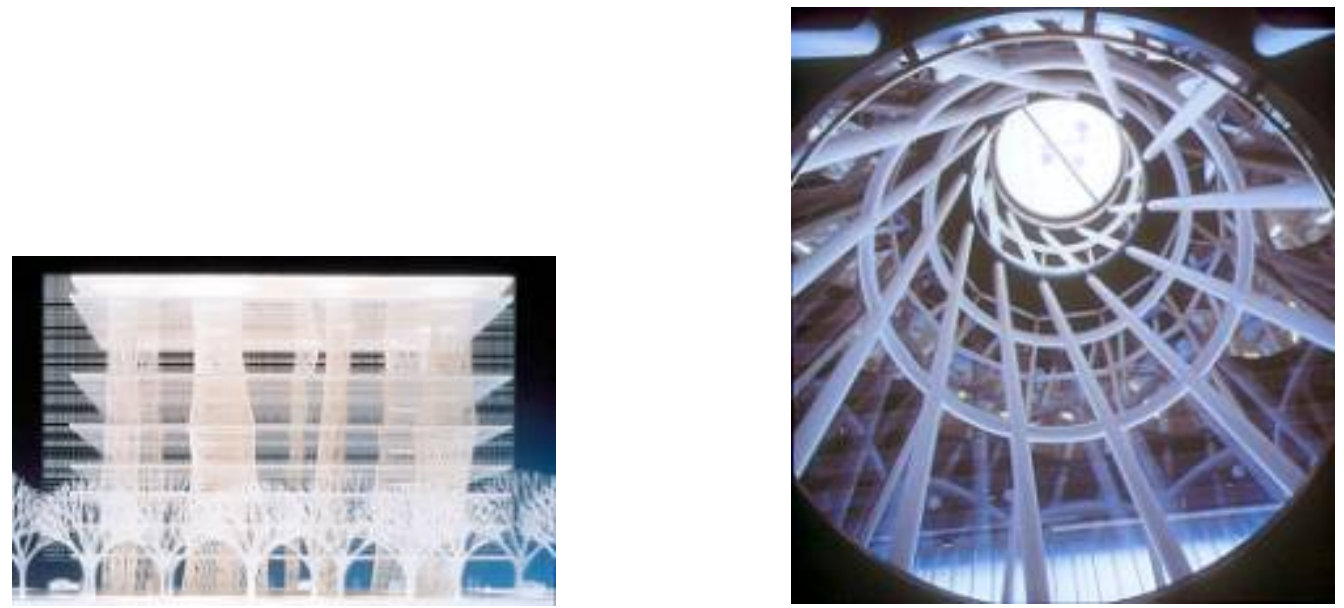

Foto 8. En el proyecto para la Mediateca de Sendai de Toyo Ito, las losas son perforadas verticalmente por doce mallas tubulares de acero. Estos túneles verticales internos se mueven a través de las losas y del espacio con una cierta autonomía en referencia al objeto.

¿Cómo se crean los hoyos? La respuesta puede ser tan simple como excavar, taladrar o esculpir, por mencionar algunas. Uno siempre hace un hoyo en (o a través de) un objeto al actuar en la materia de ese objeto. Podemos excavar en el suelo (en la superficie) en forma que se genera una discontinuidad en la lectura del campo. En este sentido el hoyo representa una discontinuidad perceptual y formal. Una identidad en la superficie la cual produce una discontinuidad. Usualmente solemos percibir un hoyo cuando percibimos una cierta discontinuidad en la superficie de objetos, en este sentido podemos establecer nuestra primera relación entre el hoyo y el artefacto arquitectónico. Como mencionamos, los hoyos son entidades superficiales, van de la mano de las superficies y en este sentido, la superficie-espacio ya no es continua en su forma general, diferentes "regiones de espacio" pueden perforan la nueva superficie. A escala arquitectónica, el hoyo puede introducir una discontinuidad cualitativa, una perturbación en la superficie que no puede ser ya leída como una abertura pero como otra cosa, como una entidad espacial consumiendo espacio. Donde, en espacio actual, el hoyo se convierte en una "cosa-en-si-misma" la cual produce una división en el "anfitrión". Para ser un individual, dentro de otras cosas, es poder distinguirse de su contexto, de su ambiente. Taladrando un objeto, destruimos parte de la -materia- del objeto, pero al mismo tiempo deformamos la consistencia de su -materia- al desplazar sus partículas para producir una condición aglomerada alrededor del nuevo individual, el hoyo. La materia dentro del hoyo se mueve de manera que provoca un incremento en la densidad en una parte del objeto. Excavar, taladrar o esculpir pueden describirse como procesos simples de deformación continua en la superficie del objeto. Perforar, punzar o punciones pueden verse como formas de corte, producen un hoyo por medio de rebanar la superficie creando un corte incompleto en la material, un hoyo. Los hoyos son casi siempre el resultado de procesos que actúan como actores primarios, mas no necesariamente, voluntarios.

¿Cómo percibimos hoyos? Como ya mencionamos, los hoyos producen una discontinuidad en la superficie del objeto donde se localiza, esta discontinuidad produce una cierta distracción hacia el ojo, hacia nuestra mente. Nuestra mente percibe un punto, un elemento extraño se mezcla en el campo de la superficie que altera completamente una lectura abierta del todo. Es esta discontinuidad que define al hoyo y le da integridad individual, nuestro modo perceptual se desliza hacia una concavidad. Desde el punto de vista fenomenológico del hoyo, podemos introducir la noción de "perturbación" o "disturbio" arquitectónico. Si hay un hoyo en una pared, podemos percibirlo gracias a que la pared tiene una cierta forma, y en hoyo depende para su existencia en la existencia de la pared, y de esa pared en particular. Una "perturbación" o "disturbio" es producido en nuestra lectura a través de la percepción de una discontinuidad en la superficie de la pared. Un hoyo como "perturbación" puede moverse o cambiar de tamaño. De la misma forma, como particular superficial, son "parasíticos" en sus anfitriones y al mismo tiempo tienen vida propia.

Si la percepción tiene que ver con materialidad, y los hoyos son cuerpos inmateriales, una teoría sobre percepción normal no aplicaría para ellos. "Si los hoyos son inmateriales, entonces no es posible reconocerlos. No pueden ni siquiera sé percibidos, ya que para que una entidad pueda ser percibida 
un flujo de causa debe de proceder de esa entidad hacia nuestro sistema sensorial. Pero ausencia de flujo casual puede originarse de algo inmaterial". Debido a esta condición inmaterial, los hoyos se convierten en abstracciones, de esta manera la percepción de abstracciones no se puede hacer de forma directa sino solamente vía la percepción de algo material del objeto o la entidad. O como Casati y Varzi argumentan, a través de la "percepción de la línea del hoyo", una percepción mediada. En este sentido, la percepción del hoyo no esta hecha a través del hoyo mismo, sino por medio de la percepción de la entidad material de donde depende (el anfitrión), de su contexto-ambiente, o de donde puede estar espacialmente relacionada de un modo relevante (el huésped). Una posible percepción de hoyos tendrá que sustentarse en la producción de una discontinuidad. Esto en la manera que un hoyo irrumpe una concepción total del objeto, ellos actúan como artefactos viscerales de distracción. Como mencionamos antes, un hoyo puede introducir una discontinuidad espacial cualitativa, un disturbio en el espacio, algo consumiendo espacio dentro de una configuración programática espacial, donde en la superficie-espacial el hoyo se convertirá en un objeto en si-mismo y el anfitrión-espacial sufre una división. En un sentido arquitectónico, en orden para ser un "individual", el hoyo, dentro de otras cosas, tiene que distinguirse de su medio-ambiente. Un hoyo puede producir un ambiente de distracción, capaz de alterar no solamente el espacio sino también la configuración programática (función) del objeto arquitectónico. El hoyo, en este sentido, tendrá que sostenerse en una cierta autonomía diferenciándose de su contenedor.

Dentro de la arquitectura, ¿que puede tomar lugar en un "hoyo"? ¿En el espacio de la nada? El potencial del hoyo como una -nueva- configuración espacial se sustenta en la cuestión de como tratar o trabajar con el concepto de la "nada", con el no-ser, lo inexistente. La falta de presencia que tradicionalmente existe en un hoyo puede darnos la idea de establecer lo "nuevo", en términos de lo que puede configurar el "anfitrión" del hoyo-arquitectónico.

\section{ARTE DE LOS HOYOS}

En los últimos cincuenta años, hemos visto en el campo de las artes una presión o preocupación hacia la representación de nada, del vacío, de lo vacante. Algunos artistas (de los cuales mencionaré algunos) han sido atraídos en el modo de trabajar con la idea del hoyo, del vacío, de la nada, básicamente concentrándose en el concepto de lo negativo en forma física. Diferentes artistas como Lucio Fontana, Donald Judd, Michael Heizer, Gordon Matta-Clark, Nancy Holt, Robert Smithson, Mary Miss y Anish Kapoor han tratado de trabajar sobre o a través del concepto de la nada ("nothingness"), de algo que esta ausente y sus modos de representación. Debido al espacio de este texto, trataré de presentar brevemente el modo de operación sobre este concepto en el trabajo de Fontana, Kapoor, Matta-Clark y Heizer ya que ellos ejemplifican en diferentes escalas esta noción, el trabajo en bastidor, en escultura, en instalación y en land-art.

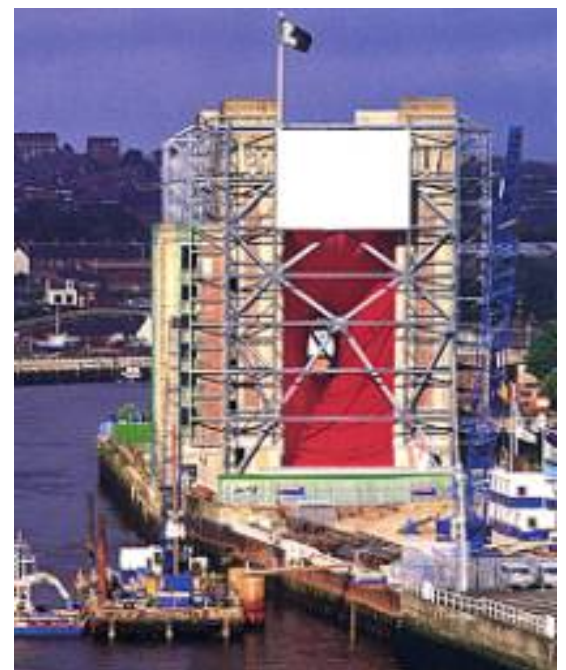

Fotos 9. El trabajo de Anish Kapoor tiende a arribar a un terreno en la mitad entre el significado del cuerpo y su ausencia. 


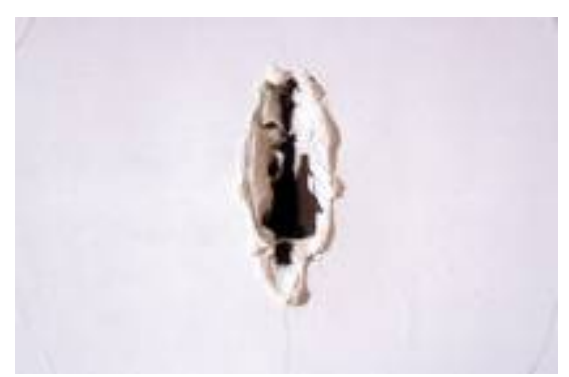

Fotos 10. Lucio Fontana se interesó en producir perturbaciones en el campo visual por medio de punzar la tela del bastidor.

Durante los cincuenta y sesenta, Lucio Fontana estaba interesado en la producción de perturbaciones en el campo visual por medio de punzar la superficie actual de trabajo (tela del bastidor). No era ya nada mas acerca de lo que se quería representar en la tela sino en una acción y locación representativa de lo que no estaba. Algo que empieza ha emerger en este trabajo es la noción del volumen implícito-, el volumen de espacio que reside entre materialidad pero sin aparecer como complemento pero como una entidad autónoma con vida propia, y que veremos mas adelante en el trabajo de Michael Heizer. El trabajo de Anish Kapoor, también, ha estado involucrado en una investigación entre lo material y lo inmaterial, entre presencia y ausencia, entre el ser y el no-ser y entre lo sólido y lo intangible. El trabajo de Anish Kapoor tiende, de cierta manera, a arribar a un terreno en la mitad entre el significado del cuerpo y su ausencia, entre estasis y actividad potencial.

Donde la "nada" se convierte en un lenguaje concreto donde a través de la creación invierte su proceso, no tiende a la exposición sino hacia las sombras en las cuales, en el final todo finalmente cae.

"La herida se abre al abismo de la nada no sirve para exorcizar el vacío, para encantarlo afuera con todo los mecanismos del arte: es a través de los labios divididos de la herida que el vacío es visible, atractivo y seductivo, prometiendo paz y ofreciendo el incomparable regalo del placer." Pier Luigi Tazzi

En relación con construcciones físicas, los últimos trabajos de Kapoor han tratado con esta polaridad dentro de la arquitectura, como en "Taran-tantara" en el Baltic Centre (2000) o en su proyecto arquitectónico «Construyendo para el Vacío" en Sevilla, España (1992).

Fuera de sus intervenciones a través de la producción de cortes y perforaciones, el trabajo de Gordon Matta-Clark estaba más interesado en la transformación del espacio estático en un ambiente dinámico por medio de estos actos. Volviendo a nuestra lectura de hoyos, la percepción de un "hoyo-espacial" es establecida por la "región de espacio" que constituye el hoyo y su relación que mantiene con el objeto donde existe. En el trabajo de Matta-Clark, el hoyo entra en conflicto con la previa interpretación legitima del objeto arquitectónico como el del común contenedor controlado. El hoyo, aquí, parece salir a la vida al presentarse como una clase de accidente en el espacio, como alguna clase de parásito el cual se puede mover en el espacio, que puede aparecer y desaparecer, y al mismo tiempo puede modificar la configuración espacial del objeto donde reside. En su trabajo "Circus" (1978) Matta-Clark trataba de introducir un sentido de confusión entre los valores del espacio positivo y negativo, de figura/fondo donde el "vacío" es el fondo, "el espacio negativo aparece chocar con espacio negativo". (Pamela Lee, Objects to be destroyed: The work of Gordon Matta-Clark, The MIT Press, 2000) El trabajo de Matta-Clark es central para una discusión posterior en hoyos, no solamente por los conceptos que mencionados arriba sino por su intrínseco proceso de creación. 

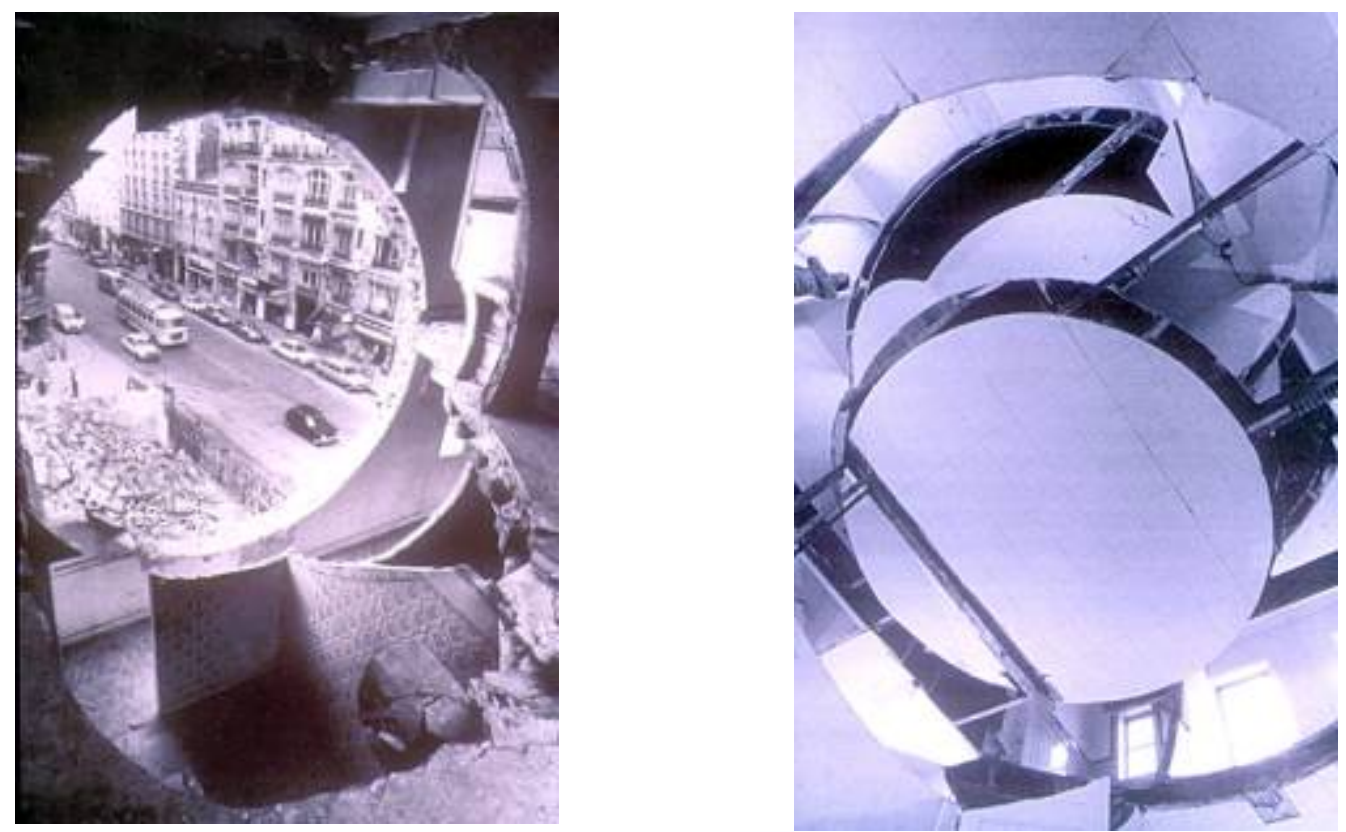

Fotos 11 y 12. En "Circus" Matta-Clark trató de introducir un sentido de confusión entre los valores del espacio positivo y

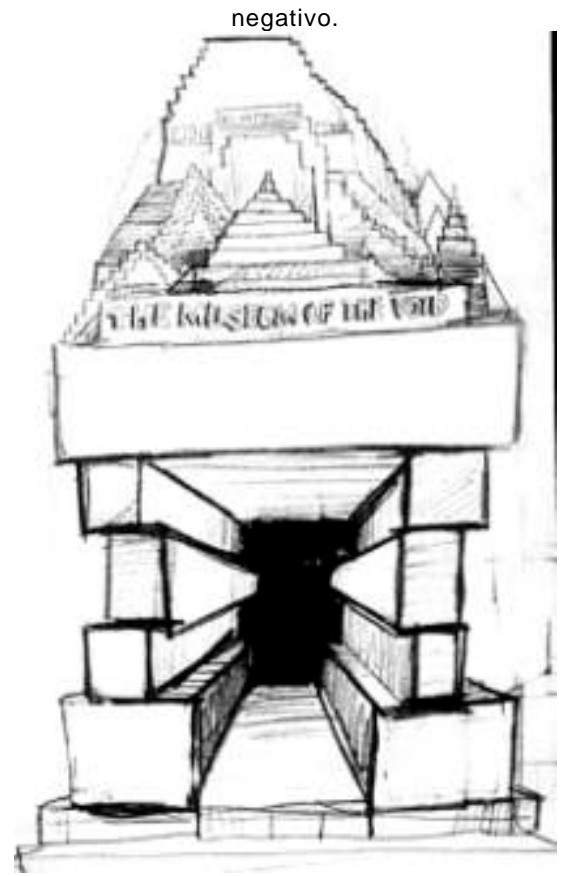

Foto 13. Robert Smithson ha denotado el proceso de excavación como una serie de obras de arte que se "desvanece" conforme se desarrolla formando "nuevos paisajes de abstracción y artífice"

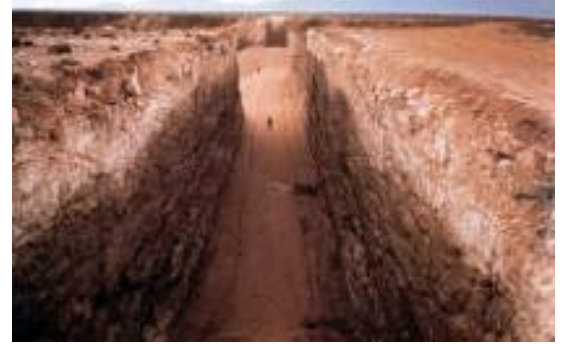


Foto 14. Michael Heizer en "Double Negative practica un corte en una pared de roca, donde el espacio abierto entre los dos cortes esta implicado, esta combinación crea un negativo doble.

Dentro del trabajo de Michael Heizer, aparte de estar relacionado con el proceso de creación, la excavación se convierte en parte física actual dentro del trabajo actuando como una entidad material, un objeto en la obra. En su ensayo "A thing is a hole in a thing it is not" (Collected Writings, 1968), Robert Smithson denota el proceso de excavación como un serie de obras de arte que se "desvanece" conforme se desarrolla formando "nuevos paisajes de abstracción y artífice". La obra de arte paisajista de Heizer, "Double Negative" (1969-70) es una entrada fundamental dentro de este argumento. Donde "fuera de ser una forma que ocupa espacio, con una superficie que delinea los limites de un volumen interno, Double Negative esta compuesto de espacio en sí mismo: es un vacío". (Earthwork and Beyond, New York, 1989) "Double Negative es un corte en una pared de roca, el espacio abierto entre los dos cortes esta implicado, esta combinación crea un negativo doble. Lo que es interesante aquí es que el centro, casi un tercio de la obra, es un volumen implícito, un volumen ausente. Su trabajo persigue la negación de la negación misma. Sus primeras obras, llamadas "Displacement Paintings", fuera de enfocarse a la formalización de relaciones entre elementos primarios como el color y la superficie, su trabajo se movía hacia la desmaterializacion: la pintura tendía hacia la ausencia o a la "dislocación" de lo material, disolviendo la realidad o la pintura- para permitirnos ver su ausencia. Lo más importante dentro de este trabajo es la sensación de vértigo en el vacío o en la nada la cual se localiza al centro de la pintura, la cual es vista desde lo negativo. Estas sacan fuera del contexto una espacialidad interior, ausente e inmaterial. Algo que reside en algún otro lado. Lo que uno debe de ver en estas obras no es tanto el perímetro, lo grueso o el color industrial, sino el centro, el lugar donde la ausencia, una forma de negatividad aparece. A través de operaciones mentales, Heizer intenta sumergirse en la búsqueda de una liga intima entre el fenómeno y el logos, una conexión a través de la expresión establecida en la definición geométrica de algo que "no se manifiesta por sí mismo": el vacío, la ausencia. Es una búsqueda de la pintura antes de la pintura. Dentro de su trabajo, el vacío y su negatividad tienen un valor positivo dentro de su operatividad interrogativa.

¿Cuál es la función de la nada? Quizás podríamos perdernos en una banal contradicción acerca del estudio de la nada, del vacío o del hoyo dentro de la arquitectura. Nuestro interés radica en una exploración que va hacia tratar de establecer una relación material del vacío. Nuestro primer contacto ha sido de cierta forma empírica, nuestro acercamiento por medio del hoyo a esta cuestión en cierta medida nos ha permitido establecer esta primera relación. La investigación apenas se abre, existe una peculiar situación hacia la búsqueda de la incógnita, en doble medida, sobre lo que esto significa. La filosofía nos lleva por el sentimiento humano acerca del pensar sobre la nada, sobre una cuestión lógica que al parecer no representa mayor problema que su propia negación. Lo que se intentó hacer con este texto fue, tratar de mover esta situación hacia algo más palpable, hacia algo tangible sin perder las implicaciones que "existen" dentro del vacío. Podemos empezar en cierta medida con los argumentos que Casati, Varzi y Koolhaas proponen, pensar negativamente, pensar a través de los hoyos no afuera de ellos. Pensemos dentro del espacio negativo, tengamos en mente la posibilidad de la existencia de lo inverso dentro de lo que podríamos considerar, y de lo que conocemos, como espacio positivo, el recinto encerrado de la vida cotidiana. Si hay una visión en el construir quizás lo inverso nos podrá ayudar a re-pensar la economía de la arquitectura, no tanto en sumar sino en construir el vacío, el hoyo.

\section{BIBLIOGRAFÍA}

BEDARD, JEAN-FRANCOIS, ED.

1994. Cities from Artificial Excavation: The Work of Peter Eisenman, 1978-1988, Canadian Centre for Architecture and Rizzoli International Publications, Montreal, Canadá.

1994. Holes and other Superficialities, The MIT Press, Cambridge, Massachusetts.

CASATI, ROBERT AND ACHILLE C. VARZI.

1999. Anish Kapoor, Fondazione Prada, Milan, Italy.

CELANT, GERMANO.

1997. Michael Heizer, Fondazione Prada, Milan, Italy.

CELANT, GERMANO.

1997. Michael Heizer, Fondazione Prada, Milan, Italy.

DAVIDSON, CYNTHIA C. ED.. 
1991. Anyone, Anyone Corporation, Rizzoli International Publications, New York.

DELEUZE, GILLES AND FELIX GUATTARI, 1987. A Thousand Plateaus, University of Minneapolis Press (trans. Briam Massumi), Minneapolis.

DISERENS, CORINNE, ED.

1993. Gordon Matta-Clark, IVAM Centre Julio Gonzalez, Valencia, Spain.

EISENMAN, PETER.

1997. "Processes of the Interstitial", El Croquis No 83, Madrid.

FONTANA, LUCIO 1899-1968, 1982. Lucio Fontana: el espacio como exploracion, Direccion General de Bellas Artes, Archivos y Bibliotecas, Madrid, Spain.

GUSSIN, GRAHAM AND ELE CARPENTER. 2001. Nothing, August Media Ltd and Northern Gallery for Contemporary Art, London.

HEGEL, G. W. F. 1975. Aesthetics, Lectures on Fine Art, Volume II, Clarendon-Oxford University Press, (trans. T. M. Knox), Oxford. 1985. Gordon Matta-Clark: A Retrospective, Museum of Contemporary Art, Chicago, III. JACOB, MARY JANE.

2000. Taratantara, Actar Publishers, Barcelona, Spain.

KAPOOR, ANISH.

1977. Disturbances, Analysis 37, pp. 147-148.

KARMO, TOOMAS.

LEE, PAMELA M. 2000. Object to be destroyed the work of Gordon Matta-Clark, The MIT Press, Cambridge, Mass.

LEWIS, DAVID K. AND STEPHANIE R. LEWIS. 1983. Holes, Philosophical Papers, Volume 1, Oxford University Press, Oxford, pp. 3-9.

MERLEAU-PONTY, MAURICE. 1962. Phenomenology of Perception. Humanities Press and Routledge \& Kegan Paul, (trans. Colin Smith), New York and London.

SARTRE, JEAN-PAUL.

1958. Being and Nothingness, Routledge, London.

1998. Anish Kapoor, Hayward Gallery Publishing, London.

TAZZI, PIER LUIGI AND HOMI K. BHABHA.

1999. Land Art, The Princeton Architectural Press, New York.

TIBERGHIEN, GILLES A.

WHITFIELD, SARAH. 